\title{
A numerical study of the effect of bolt thread geometry on the load distribution of continuous threads
}

\author{
Abdulla Sherif Mahmoud Fathalla*, Ali Akhavan Farid*****, Reza Moezzi***** and Seyed \\ Saeid Rahimian Koloor** \\ * Department of Mechanical, Materials and Manufacturing Engineering, University of Nottingham Malaysia, 43500, Semenyih, Malaysia. \\ ** Institute for Nanomaterials, Advanced Technologies and Innovation, Technical University of Liberec, Czechia. \\ *** Faculty of Mechatronics, Informatics and Interdisciplinary Studies, Technical University of Liberec, Czechia. \\ **** Corresponding Author: ali.farid@nottingham.edu.my
}

Submitted :24/02/2020

Revised :09/03/2021

Accepted : 25/04/2021

\begin{abstract}
Load distribution has been studied extensively for ISO thread, but the load distribution on power screw threads, specifically ACME and Square threads, has not been studied yet. In this article, axisymmetric two-dimensional and three-dimensional Finite Element Analyses have been conducted on bolts with different sizes and thread geometries to examine the effect of the thread geometry on the load distribution. The thread geometries were studied with ISO, ACME, and Square threads attention. The sizes used are from the ISO coarse series. In order to investigate on the effect of bolt thread geometry, several simulations have been performed. The two-dimensional simulation results have shown reliable performance in determining the load distribution behavior when the thread geometry is modified. Moreover, the results agreed with the three-dimensional simulation outcomes regarding the load distribution behavior when the size is varied.
\end{abstract}

Keywords: Bolt thread geometry; Load distribution; Finite element simulation; ISO; ACME; Square threads.

\section{INTRODUCTION}

Threads are used in fastenings like screws and bolts, and in addition, they deliver a linear motion drive for power transmission (Carvill, 1993). Evaluation of the stress behavior associated with these threads is the key design concept in mitigating their failure in critical situations. Bolts have been known to fail at the root of the first thread, meaning that the load distribution along the threads of the bolt is uneven and the first thread carries most of the load (Den Hartog, 1929). Several ways have been used to examine the load and stress distribution, from theoretically in (Den Hartog, 1929), experimentally in (Goodier, 1940), to finite element (FE) analysis in (Maruyama, 1974). The FE simulation has been recognized as an effective and important method for design, modelling and analysis of complex materials and structure in various industrial applications (Koloor, 2011, 2019, 2020). All the previously published articles concentrate on either ISO threads or nonstandardized threads geometries. There are a variety of thread forms that are used in different applications, which involve the same behavior of load and stress distribution. The interest 
in the behavior of bolts under load was initiated by Den Hartog (Den Hartog, 1929) when they investigated the stresses in turbine blade bolts to discover that the load distribution is not uniform since most bolts fail at the root of the first thread. Then, they derived an equation to hypothetically calculate the theoretical load distribution in ACME thread. Goodier (Goodier, 1940) was the first to investigate this behaviour using experiments. They measured the radial expansion and the axial compression of the steel nuts using extensometers to obtains the stresses and the load distribution in the threads. In addition to stress and load distribution analysis, tensile testing also can be handled to obtain the tension (Dhoska, K., 2019). Hetenyi (Hetenyi, 1943) later conducted an experiment to examine the behavior using photo elastic stress-freezing method. Sopwith (Sopwith, 1948) developed a theory to calculate the force on each thread. According to Sopwith results, the number of threads engaged affects the load distribution when the bolt is under load, but that is only up to a certain limit. When the limit is reached, the load distribution remains the same for the leading threads. They also proposed that the coefficient of friction between bolt and nut $(\mu)$ affects the load distribution. The higher the $\mu$, the larger load concentration at the leading threads. They also compared their findings with the findings of Goodier (Goodier, 1940) and Hetenyi's (Hetenyi, 1943) experimental results and found that their results were reasonably accurate. Sopwith's method restricts the calculation to the same material for both the nut and bolt. However, this is not the case for real life scenarios. Different material could be used to resolve problems such as corrosion, large load concentration, or seizing (Sato, Yamamoto, Li, \& Ando, 2008).

The use of FE simulation did not start until the 70th decade, and at the time, only 2D FE analysis had been used. The quality of results from FE simulation during that time however is questionable. One of the first experiments to use FE analysis was conducted by Maruyama (Maruyama, 1974). Although they were accurate to some extent, the results were unreliable due to the unfamiliarity with FE analysis at the time. They blamed the inaccuracies on the high stress concentration at the root, the concertation is hard to simulate due to the primitive meshing techniques (Maruyama, 1974). Bretl and Cook (Bretl \& Cook, 1979) attempted to use 2D FE analysis to determine the distribution as well. The results they managed to obtain were exceptionally close to Sopwith's theoretical results and were the most accurate results at the time considering most FE analysis results at the time were erroneous (Bretl \& Cook, 1979). This result was achieved by using a mesh with quadrilateral elements that contained orthotropic properties, which are linked to the properties of the material used (Kenny \& Patterson, 1985). Bretl and Cook (Bretl \& Cook, 1979) also optimized the boundary conditions to better mimic reality. The elements used on the supporting edges of their model of the nut did not affect the radial movement of the nut.

Figure 1 shows the results of different experiments' load distribution against distance from the loaded surface of the nut. Bretl and Cook's (Bretl \& Cook, 1979) experiment results mostly agree with Sopwith's (Sopwith, 1948) theory and are considered the most accurate FE analysis of its time. Later, Yamamoto (Yamamoto, 1980) could obtain the theoretical load distribution on an axially loaded using the theory of elasticity, where the thread is simplified as a cantilever model of an isosceles trapezoid. Yamamoto's theory allowed the calculation of the load distribution for different materials of the bolt or nut and the most used analytical method of obtaining the load distribution. In late 20 century, Draoni (Dragoni, 1994) studied the effect of $\mu$ on the stress concentration of the leading threads of the bolt. Their 2D FE simulations revealed that the stress concentration increases with the increase of $\mu$, as illustrated in Figure 2, up to a certain point; after that, stress concentration becomes independent of $\mu$. In another study (Dragoni, 1997), the authors found that endurance strength of the bolt can be enhanced about $40 \%$ by finding the optimal pitch value. 


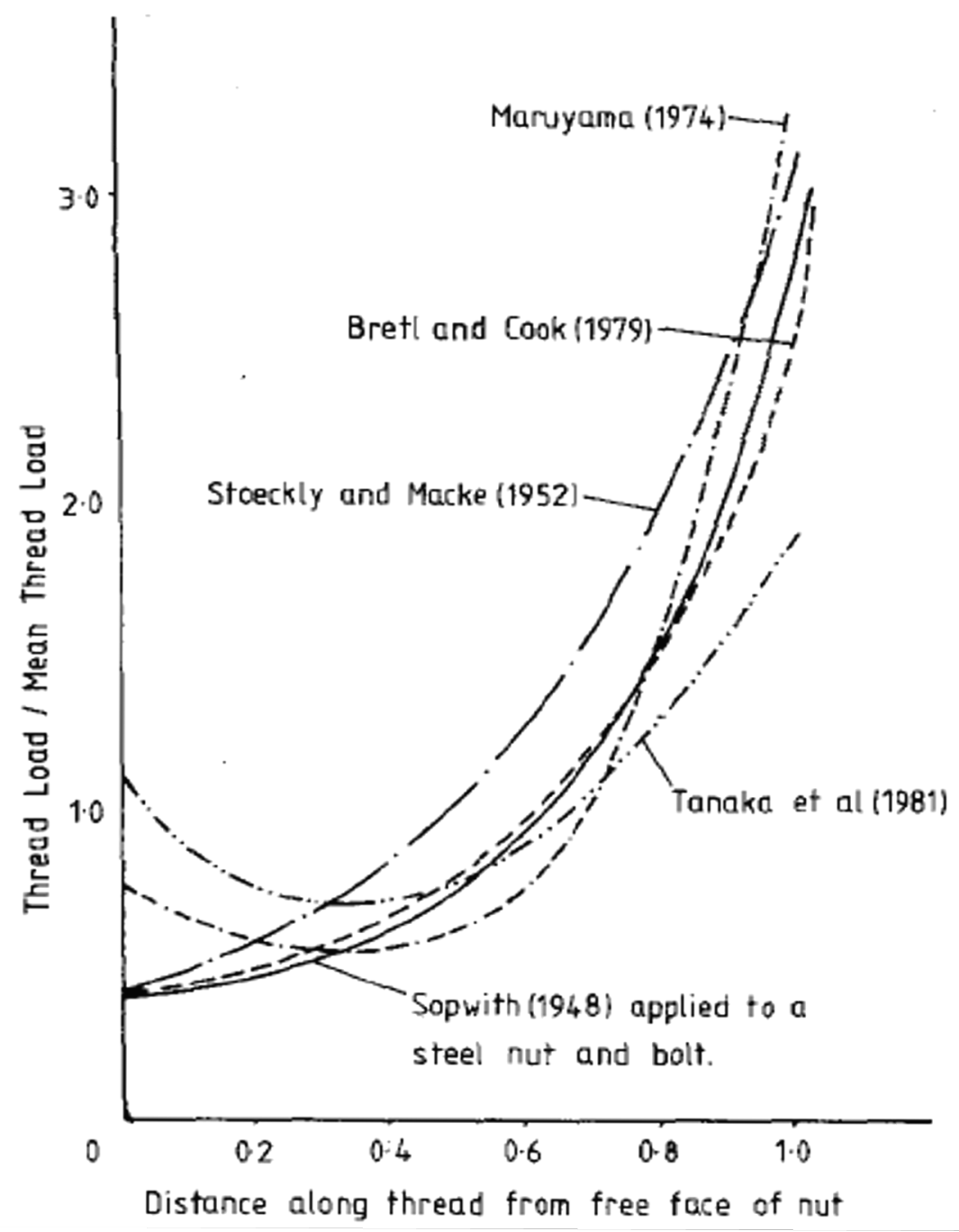

Figure 1. Load distribution results comparison (Bretl \& Cook, 1979).

Chen and Shih's (Chen \& Shih, 1999) publication was one of the first articles to use 3D FE simulation. Their paper aimed to study the helical effect on the simulation by simulating an axisymmetric 2D and 3D model. They also varied $\mathrm{P}$ (thread pitch) to analyze the load distribution. Their results indicate that using a smaller P resulted in a more even load distribution. But the smaller $\mathrm{P}$ resulted in a larger difference between the 2D and the 3D model. They also pointed out that Yamamoto's analytical result underestimated the load on the leading threads. Yang et al (Yang, et al., 2013) also investigated the load distribution. They involved varying $\mathrm{P}$ and $\mathrm{E}$ of the joint to study their effect on the load distribution using 2D and 3D FE analysis. Results showed that the smaller the ratio of $\mathrm{E}$ of the nut to the $\mathrm{E}$ of the bolt, the more uniform the load distribution along the threads. Their results also conclude that the thread pitch aids in the load distribution. Zhang et al. (Zhang, Gao, Niu, \& Liu, 2018) developed a new analytical method for evaluation the load distribution, which follows the 3D FE analysis findings more closely. This addresses the difference between the analytical and simulation values found in (Chen \& Shih, 1999) as well. Their analytical method also allows for full customizability of the thread geometry shape. 


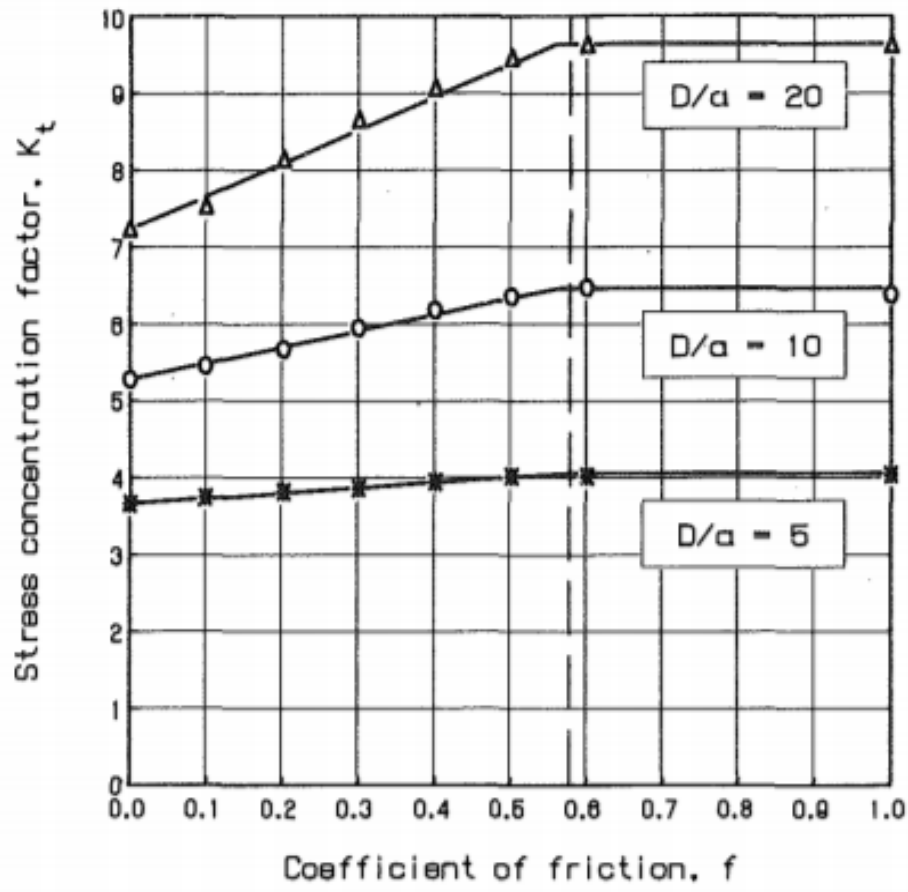

Figure 2. First thread stress concentration versus $\mu$ (Dragoni, 1994) (D/a is Nominal diameter and P ratio).

In recent studies, Nie et al. (Nie, 2018) evaluated load distribution of standard thread in the concretion structure of a TBM butterhead through experimental and finite element analysis. In their study, uneven distribution of axial force was recognized the main contributor of the bolt fracture when it was loaded under axial static and dynamic loads. They also found out that $25.1 \%$ to $28.1 \%$ of the total load will concentrate near the root of the first thread. However, it is shown that this percentage will increase at elevated temperatures, while Zhou et al. (Zhou et al., 2015) carried out experimental and FE studies to evaluate load distribution in ISO threads of porous metal-ceramic composite at temperature of $1200^{\circ} \mathrm{C}$. They reported that $74 \%$ of the load was carried by the first thread at such severe condition. There is another interesting study (Wang et al., 2020), which investigated the effects of uniform temperature and bolts fracture on the behavior of high strength bolted frictional steel connection. This research has provided useful reference for the frictional steel connection design of high strength bolts. Coria et. al (Coria, et al., 2020) developed a semi-analytical model to predict the load distribution of various pitch of ISO thread which has been used to find the optimum thread spacing for achieving the most uniform load distribution on the threads. The effect of washer on stress distribution of ISO thread was studied by Brutti (Brutti et al., 2017) through finite element modelling, where they claimed that the load concertation on the first thread can be reduced by enlarging the internal diameter of the washer.

The study of load distribution of bolt threads has extended in the application of dental implant thread in recent years. Geramizadeh et al. (Geramizadeh, et al., 2018) found the optimum depth and pitch of V-shaped dental thread through FE analysis and response surface optimization resulted optimal stress distribution. In another study, Finite element method was also applied to find the optimum treading angle. It was concluded that an angle of 45 degrees leads to the most even stress distribution on threads (NarendraKumar, et al., 2018).

All studies mentioned above were aimed towards analyzing the stress and load distribution of ISO threads with the major thread angle of $60^{\circ}$. However, FE analysis has never been conducted to examine the load distribution of 
different thread types such as Square or ACME as the most common type of thread shape in power screws. Therefore, this study aimed to examine the difference in the load distribution behavior between ISO, ACME, and Square thread using axisymmetric 2D and 3D simulation.

All recent mentioned research studies were aimed towards analyzing the stress and load distribution of ISO threads with a thread angle of $60^{\circ}$ however FE analysis has never been conducted for different thread types such as Square or ACME to examine the load distribution. Therefore, this article mainly aims to examine the difference in the load distribution behavior between ISO, ACME, and Square thread using axisymmetric 2D and 3D simulation which have revealed novel relationships between thread parameters that were not considered in earlier research studies.

\section{METHODOLOGY}

From the wide range of choices, three sizes from ISO coarse series are selected. D (thread major diameter) and $\mathrm{P}$ (thread pitch) are kept constant for each size and emulated into ACME and Square thread profiles (according to Figure 3).

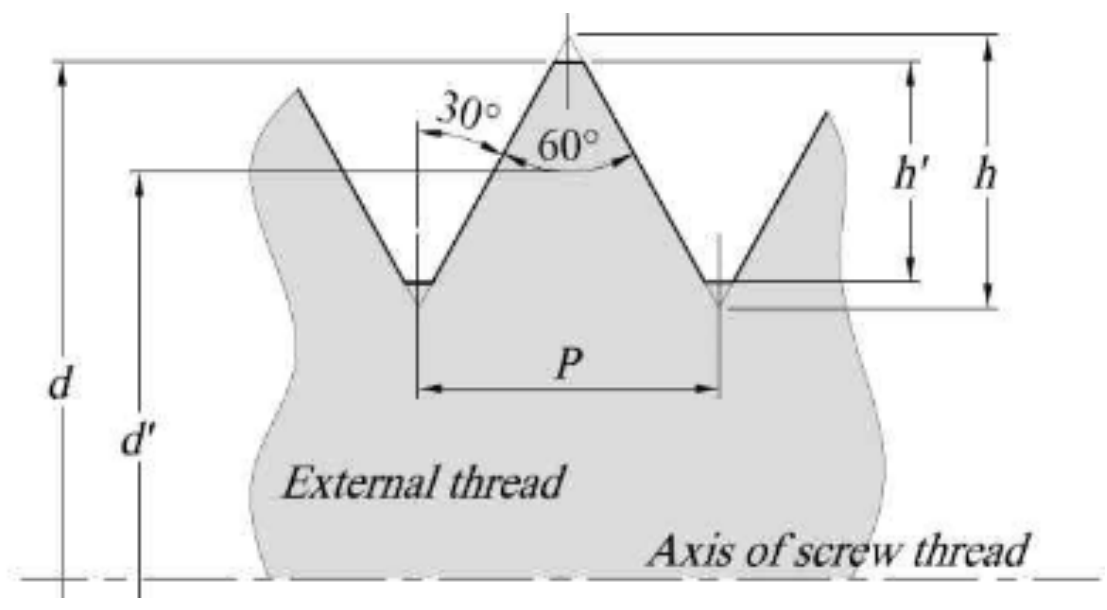

Figure 3. Standard design parameters of threads (Lee, 2016).

Table 1. Constants values for simulation.

\begin{tabular}{|c|c|}
\hline Constants & Value \\
\hline $\mathbf{E}$ (Young Modulus) & $200 \mathrm{GPa}$ \\
\hline $\boldsymbol{v}$ (Poisson's ratio) & 0.3 \\
\hline $\boldsymbol{\mu}$ (Friction Coefficient) & 0.2 \\
\hline
\end{tabular}


The difference in load distribution behavior by changing the size has also been analyzed. All the other parameters are kept constants for all the simulations; this includes the ratio of cross-sectional areas of the bolt and nut, E (Young Modulus) which is set to $200 \mathrm{GPa}, \mu$ (Friction Coefficient) which is set to 0.2 , and $v$ (Poisson's ratio) to 0.3 . These values are common for such FE simulations and have been used before in previous studies. The $2 \mathrm{D}$ and 3D simulations in (Chen \& Shih, 1999) were replicated successfully with very minimal difference. All the simulations were conducted on ANSYS software, the frictional contact behavior of Symmetric, and the formulation of Normal Lagrange was used. The standard ISO, Square, and ACME thread geometries were used, class 2 tolerance was used for ACME thread (ISO - International Organization for Standardization, 2014) (Beardmore, 2013) (Engineers Edge, n.d.). Engagement is maintained between eight threads for all the simulations. Table 2 indicates the sizes simulated along with the force used for each size; the force, however, is irrelevant since the load distribution is a ratio of the total force and independent from it (Zhang, Gao, Niu, \& Liu, 2018).

Table 2. Thread sizes to use for simulation.

\begin{tabular}{|c|c|c|}
\hline $\mathbf{D}(\mathbf{M M})$ & $\mathbf{P}(\mathbf{M M})$ & FORCE $(\mathbf{N})$ \\
\hline $\mathbf{5}$ & 0.8 & 1000 \\
\hline $\mathbf{3 0}$ & 3.5 & 6000 \\
\hline $\mathbf{5 6}$ & 5.5 & 11200 \\
\hline
\end{tabular}

\section{D Model}

The modelling of the 2D axisymmetric model was done on Design Modeller in ANSYS. Figure 4a illustrates the model used to carry out the $2 \mathrm{D}$ axisymmetric simulation. The highlighted regions indicate the regions of contact. A force is applied to the top edge of the bolt (pointing upwards), the top edge of the nut is constrained in the y-axis direction, and both vertical edges of the bolt and nut are constrained in the x-axis direction to prevent thread disengagement (Figure 5). This model is replicated for each variation of thread geometry and size. Quadrilateral mesh is used where its size was defined by length-to- width ratio of 1.0, and finer mesh were used at high stress areas (the root of bolt and nut threads). Figure $4 \mathrm{~b}$ illustrates an example of the mesh used to conduct the $2 \mathrm{D}$ axisymmetric simulation.
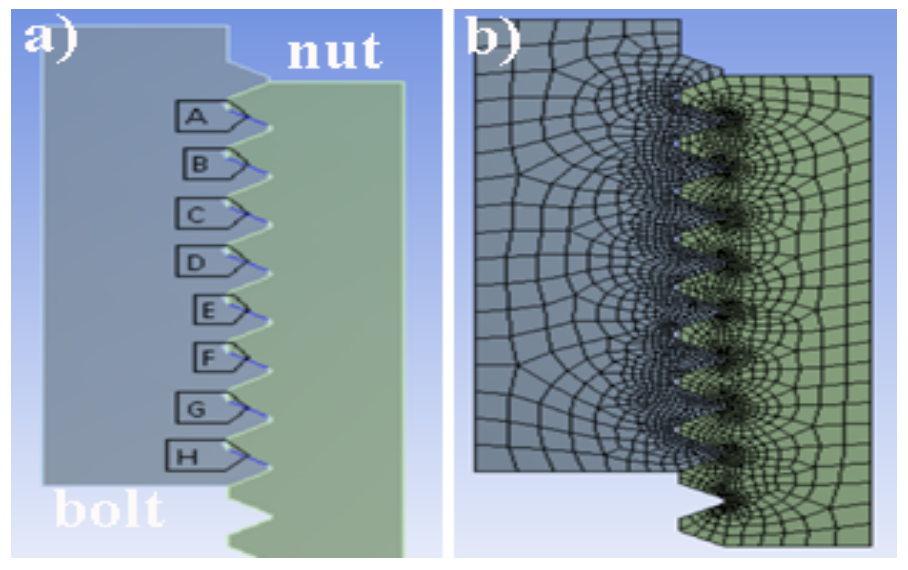

Figure 4. a) 2D axisymmetric model of bolt and nut (ISO), b) 2D axisymmetric model mesh. 


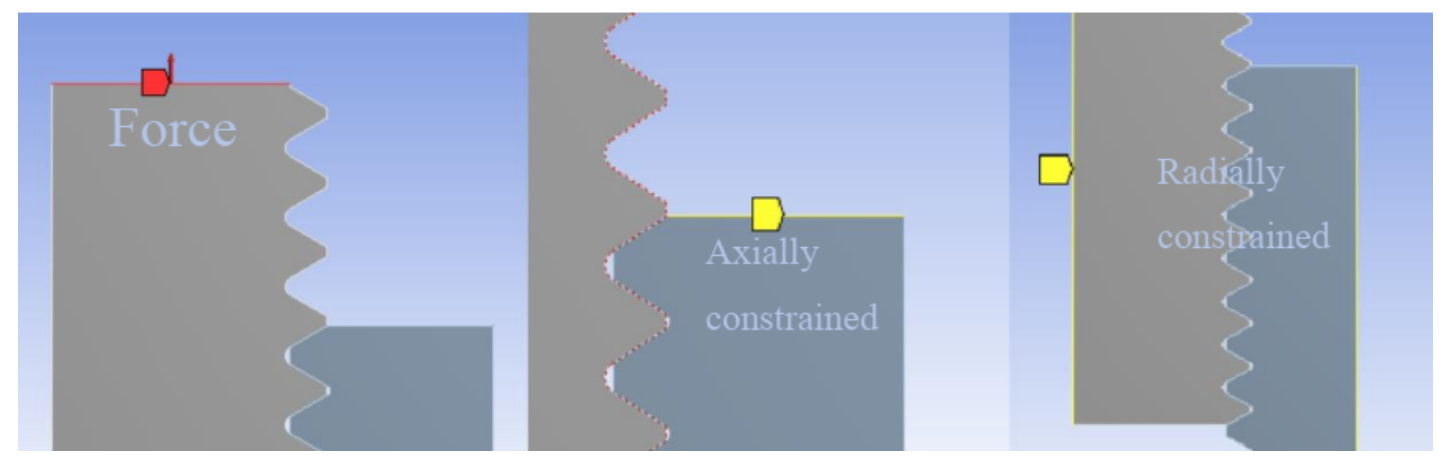

Figure 5. Boundary conditions in the 2D FE simulation.

\section{D Model}

The 3D model was developed in SpaceClaim in ANSYS. Figure 6a shows the 3D model of bolt and nut. A cylinder is extruded first, and then the appropriate thread geometry is helically extruded around the cylinder for the height of $\mathrm{P}$ eight times to create a bolt. Each of the eight threads is helically extruded individually to obtain the load carried by each one. The nut body and thread are both helically extruded together around the bolt threads eight times as evident in Figure 6b. This allows for full thread engagement between the bolt and nut threads at all threads.
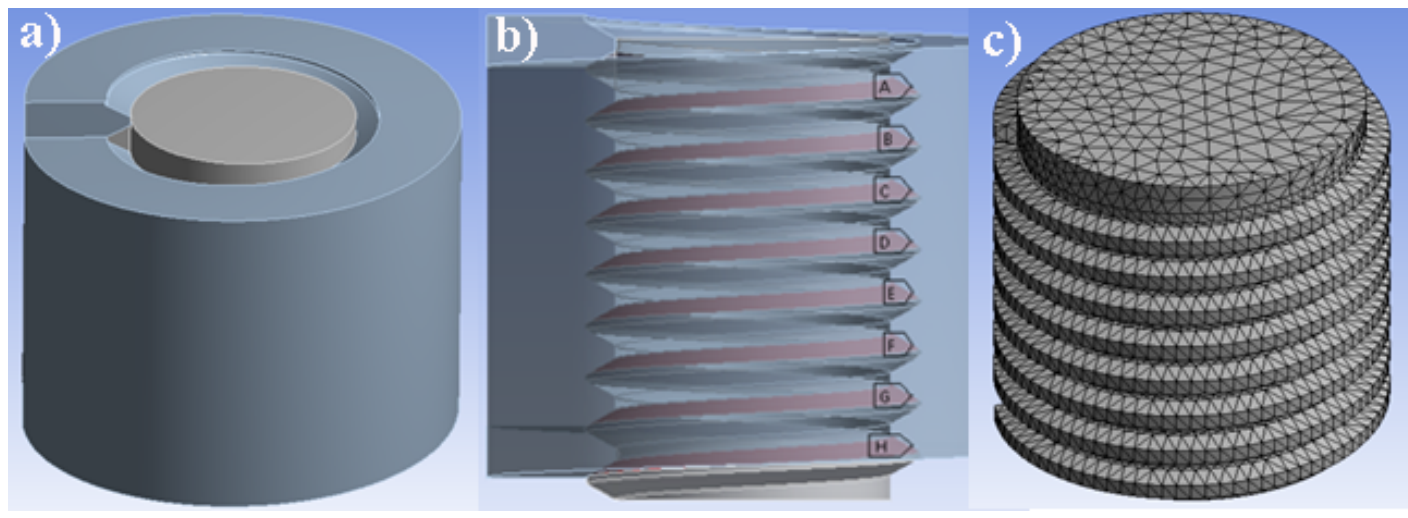

Figure 6. a) 3D model of Bolt (grey) and nut (blue) (ISO), b) 3D simulation of contact surfaces, c) 3D model mesh (ACME).

Similar to the 2D model, contact is defined only in the direction of the force for all of the eight threads as evident in Figure $6 \mathrm{~b}$. A force of $1 \mathrm{kN}$ (pointing upwards) is applied on the bolt body surface. The top surface (loaded surface) of the nut was constrained axially and tangentially. The nut is not constrained radially since this will affect the load distribution. The bolt' $\mathrm{s}$ loaded surface was constrained tangentially to prevent the bolt from rotating due to the helical effects of the threads (Figure 7). These conditions are replicated for all the thread geometries and sizes. Through mesh-sensitivity analysis, tetrahedral mesh with size of $0.0015 \mathrm{~m}$ was selected for the component, while meshes were refined at high stress areas (the root of bolt and nut threads). Figure $6 \mathrm{c}$ illustrates an example of the mesh used. 


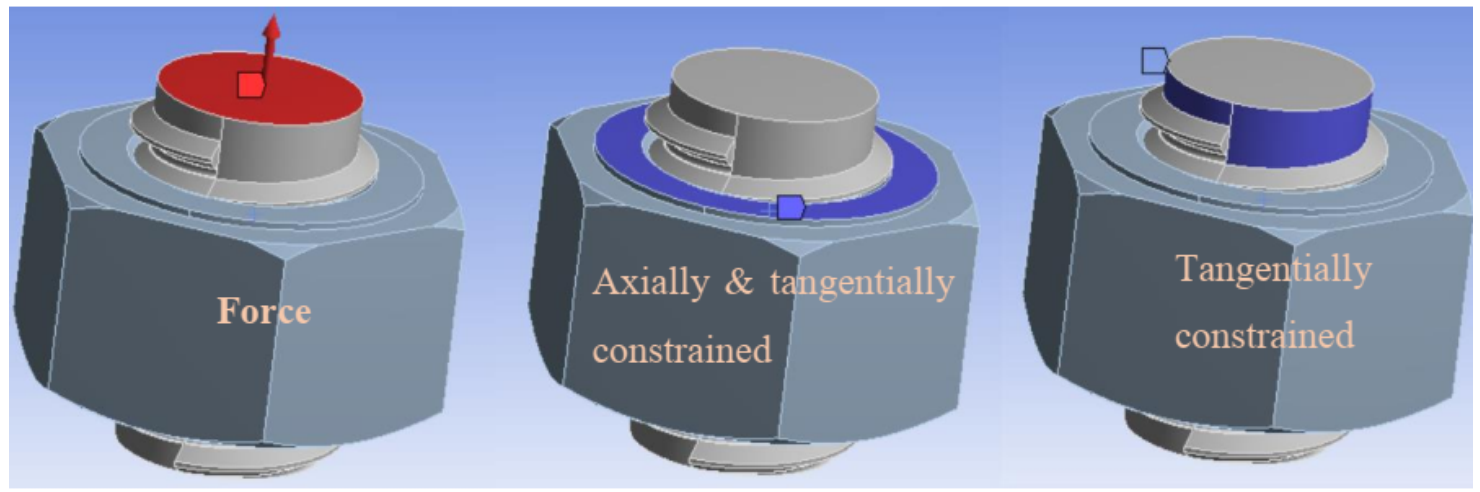

Figure 7. Boundary conditions of 3D FE simulation.

\section{RESULTS \& DISCUSSION}

The first two sizes in Table 2 were considered with three different thread geometries: ISO, ACME, and square. The results were studied for the following trends.

\section{Verification of the Finite Element Model}

The verification process is performed through proper comparison of the computational work with experimental counterpart in the published literature, as recommended in the literature (Koloor, 2018, 2020; Theng, 2017; Karimzadeh, 2019). Similarly, to verify the developed FEM and constants used in this work, a verification simulation was conducted by using the force and thread geometry used by Chen et al. (Chen \& Shih, 1999). They have also verified their analysis by comparison between their results and Yamamoto's method (Yamamoto, 1980). Table 3 illustrates the difference between the load distribution obtained from the reference and the distribution obtained from the simulation. The percentage difference between the two load distribution values is satisfactory for the leading threads, especially the first three threads where the load is concentrated. The percentage difference is exceptionally high at the last two threads. This is due to the low load carried by these threads.

Table 3. The comparison between the results of the reference and developed model.

\begin{tabular}{|c|c|c|c|}
\hline Thread number & Reference load (lbf) & Simulation load & Perc. Diff \\
\hline 1 & 1699.00 & 1709.40 & $-0.61 \%$ \\
\hline 2 & 1039.00 & 1037.30 & $0.16 \%$ \\
\hline 3 & 721.90 & 742.26 & $-2.82 \%$ \\
\hline 4 & 530.10 & 555.16 & $-4.73 \%$ \\
\hline 5 & 405.40 & 418.78 & $-3.30 \%$ \\
\hline 6 & 322.90 & 318.47 & $1.37 \%$ \\
\hline 7 & 261.80 & 239.94 & $8.35 \%$ \\
\hline 8 & 180.10 & 138.71 & $22.98 \%$ \\
\hline
\end{tabular}




\section{D Analysis}

Figure 8 illustrates the contour of the von Mises stress of the axisymmetric 2D simulations, providing an overview of the stress distribution. Stress is highest at the root with the highest being at the root of the first thread as observed by (Den Hartog, 1929).
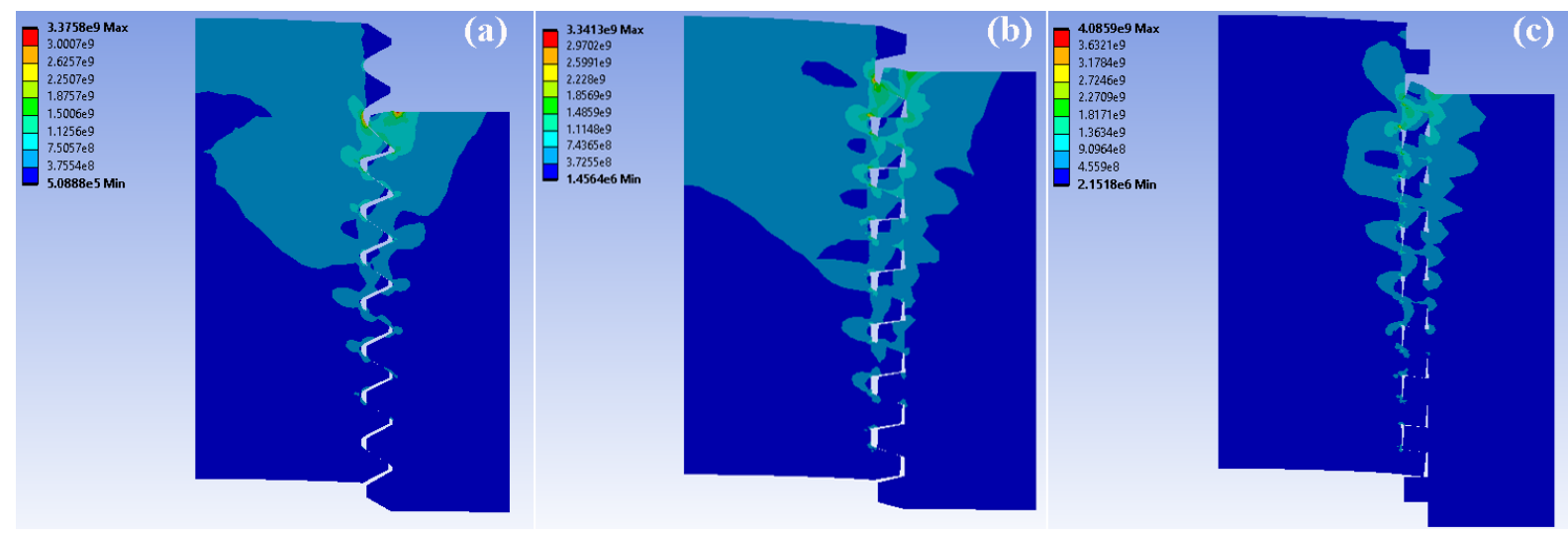

Figure 8. 2D FE simulation result of Von Mises stress contour of D=30 mm; a) ISO, b) ACME, c) Square.

Table 4 displays the 2D axisymmetric FE simulation results. The 2D axisymmetric usually underestimates the load on the leading threads of the bolt (Chen \& Shih, 1999). 3D simulations are inherently more accurate since they take the helical effect of threads into account (Chen \& Shih, 1999). The difference between 2D and 3D simulations' load distribution varied significantly when $P$ is varied (Chen \& Shih, 1999). Simulation of 5 and $30 \mathrm{~mm}$ indicated that 2D model failed to show the significance of geometry effect on the load distribution, which is due to the contribution of helical effect in the load distribution being neglected in the 2D simulation (Tan, et al, 2020). That is why simulations were not conducted for $\mathrm{D}=56 \mathrm{~mm}$. However, a trend was observed when size was varied in all the thread types.

Table 4. Load distribution of threads in 2D simulation.

\begin{tabular}{|c|c|c|c|c|c|c|}
\hline \multicolumn{7}{|c|}{ Load distribution (\%) } \\
\hline \multirow{2}{*}{$\begin{array}{c}\text { Thread } \\
\text { Tumber }\end{array}$} & \multicolumn{3}{|c|}{$2 \mathrm{D} 5 \mathrm{~mm}$} & \multicolumn{3}{c|}{$2 \mathrm{D} 30 \mathrm{~mm}$} \\
\cline { 2 - 7 } & ISO & ACME & Square & ISO & ACME & Square \\
\hline 1 & $32.4 \%$ & $32.5 \%$ & $29.6 \%$ & $28.2 \%$ & $26.4 \%$ & $25.8 \%$ \\
\hline 2 & $20.4 \%$ & $20.0 \%$ & $20.0 \%$ & $18.2 \%$ & $16.9 \%$ & $17.9 \%$ \\
\hline 3 & $14.9 \%$ & $15.5 \%$ & $15.1 \%$ & $14.4 \%$ & $14.6 \%$ & $14.6 \%$ \\
\hline 4 & $11.0 \%$ & $11.8 \%$ & $11.5 \%$ & $11.9 \%$ & $12.4 \%$ & $12.3 \%$ \\
\hline 5 & $8.2 \%$ & $9.1 \%$ & $8.8 \%$ & $9.8 \%$ & $10.5 \%$ & $10.3 \%$ \\
\hline 6 & $6.1 \%$ & $7.1 \%$ & $6.8 \%$ & $8.0 \%$ & $8.7 \%$ & $8.6 \%$ \\
\hline 7 & $4.5 \%$ & $5.6 \%$ & $5.1 \%$ & $6.1 \%$ & $6.5 \%$ & $6.7 \%$ \\
\hline 8 & $2.5 \%$ & $4.0 \%$ & $3.1 \%$ & $3.3 \%$ & $3.7 \%$ & $4.0 \%$ \\
\hline
\end{tabular}


As illustrated in Figure 8, the three leading threads carry less load, while the rest of the threads carry more when the size is increased. A difference of $4 \%-6 \%$ of load on the first thread is observed when the size is changed, this change of load distribution behaviour can be attributed to disproportionate increase in $\mathrm{D}$ and $\mathrm{P}$ when size is varied since varying $P$ has a significant effect on load distribution (Yang, et al., 2013).

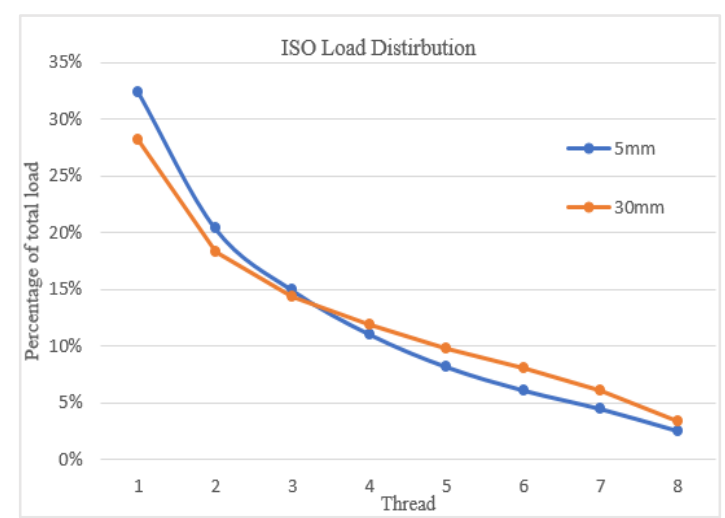

(a)

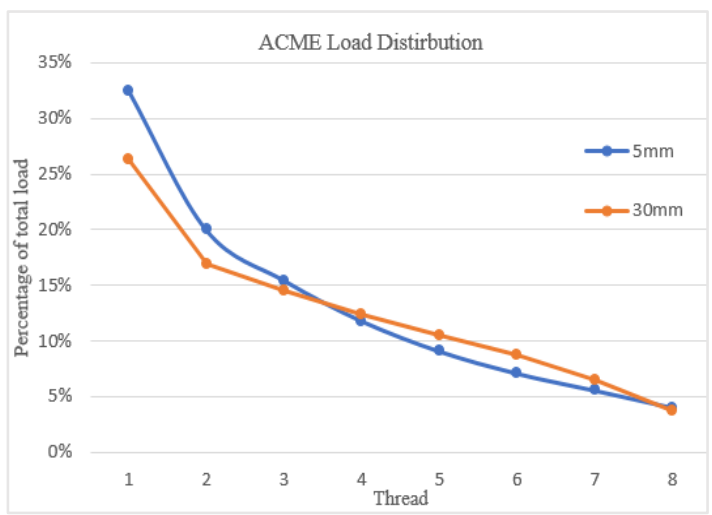

(b)

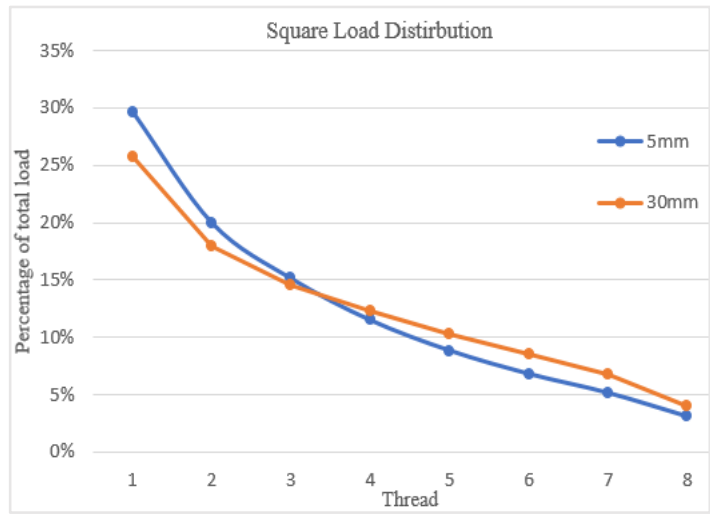

(c)

Figure 8. Effect of size on load distribution (2D), a) ISO, b) ACME, c) square.

\section{D Analysis}

Figure 9 illustrates the contour of the von Mises stress of the 3D simulations with the same variables used in 2D. Stress is highest at the root with the highest being at the root of the first thread like what is observed in the 2D simulations. 

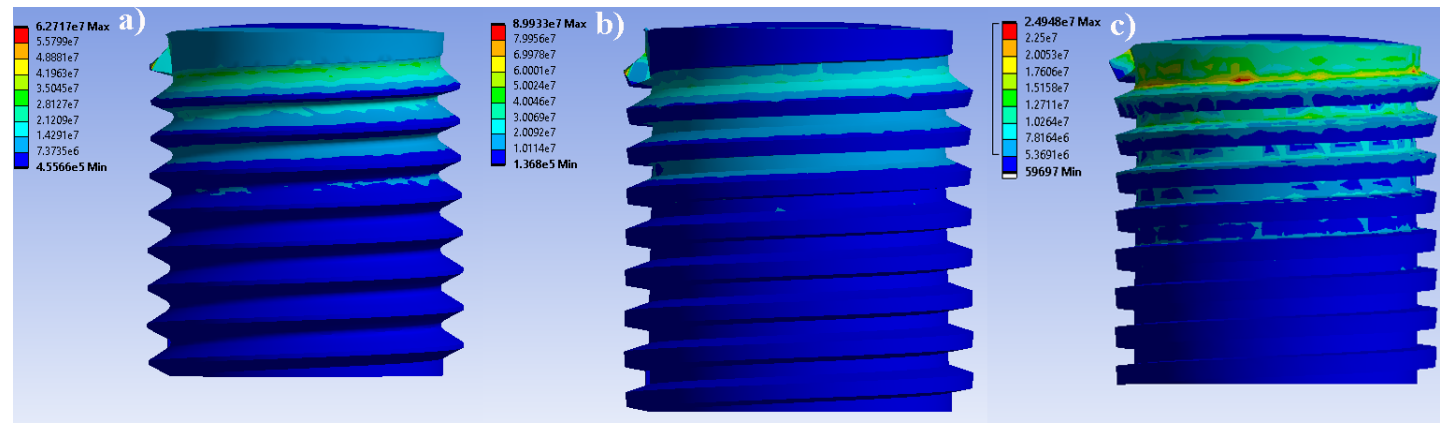

Figure 9. Von Mises contour (3D), a) ISO, b) ACME, c) square.

Table 5 demonstrates the results obtained from the 3D FE simulations. Unlike the 2D simulations, there is a significant trend in load distribution behavior when the thread geometry is changed as evident in the following figure 10.

Table 5. Load distribution of threads in 3D simulation.

\begin{tabular}{|c|c|c|c|c|c|c|c|c|c|}
\hline \multicolumn{3}{|c|}{ Load distribution (\%) } \\
\hline \multirow{2}{*}{$\begin{array}{c}\text { Thread } \\
\text { number }\end{array}$} & \multicolumn{3}{|c|}{$3 \mathrm{D} 5 \mathrm{~mm}$} & \multicolumn{3}{c|}{$3 \mathrm{D} 30 \mathrm{~mm}$} & \multicolumn{3}{c|}{$3 \mathrm{D} 56 \mathrm{~mm}$} \\
\cline { 2 - 11 } & ISO & ACME & Square & ISO & ACME & Square & ISO & ACME & Square \\
\hline 1 & $41.9 \%$ & $35.6 \%$ & $32.3 \%$ & $36.2 \%$ & $30.7 \%$ & $27.1 \%$ & $33.8 \%$ & $28.7 \%$ & $25.3 \%$ \\
\hline 2 & $22.3 \%$ & $22.0 \%$ & $21.8 \%$ & $20.7 \%$ & $20.9 \%$ & $19.9 \%$ & $19.8 \%$ & $19.6 \%$ & $19.5 \%$ \\
\hline 3 & $13.2 \%$ & $14.9 \%$ & $15.3 \%$ & $13.6 \%$ & $14.9 \%$ & $15.4 \%$ & $13.6 \%$ & $14.9 \%$ & $15.2 \%$ \\
\hline 4 & $8.4 \%$ & $10.0 \%$ & $10.7 \%$ & $9.4 \%$ & $10.9 \%$ & $11.9 \%$ & $9.9 \%$ & $11.4 \%$ & $12.2 \%$ \\
\hline 5 & $5.5 \%$ & $6.9 \%$ & $7.7 \%$ & $6.8 \%$ & $8.1 \%$ & $9.2 \%$ & $7.5 \%$ & $8.4 \%$ & $9.7 \%$ \\
\hline 6 & $3.8 \%$ & $4.9 \%$ & $5.6 \%$ & $5.1 \%$ & $6.2 \%$ & $7.2 \%$ & $5.9 \%$ & $7.0 \%$ & $7.9 \%$ \\
\hline 7 & $2.9 \%$ & $3.6 \%$ & $4.1 \%$ & $4.2 \%$ & $4.9 \%$ & $5.5 \%$ & $5.0 \%$ & $5.8 \%$ & $6.2 \%$ \\
\hline 8 & $2.5 \%$ & $2.5 \%$ & $2.9 \%$ & $4.0 \%$ & $3.3 \%$ & $4.0 \%$ & $4.6 \%$ & $4.0 \%$ & $4.2 \%$ \\
\hline
\end{tabular}

The 3D FE simulation results clearly illustrate that the first thread of the ISO thread geometry carries a higher portion of the load than ACME and Square thread geometries, while the first thread of ACME thread geometry carries a higher portion of the load than Square thread geometry. This trend is consistent throughout all the sizes as evident in Figure 10. This trend follows the trend suggested by Zhang et al.'s (Zhang, Gao, Niu, \& Liu, 2018) proposed theory. The second thread carries approximately the same portion of the load for all thread geometries while the remainder of the load is spread out on the rest of the threads. Several differences in the geometry can contribute to this trend. The length of base of the thread, the height of the thread, and the thread angle are factors that change the second moment of area which have a significant effect on the load distribution (Zhang, Gao, Niu, \& Liu, 2018).

The difference of the load carried by the first thread for the three thread geometries is very close for the three sizes, meaning that the difference between load carried by ISO and ACME threads for the three sizes is very close; this is true for all other threads and sizes. The difference between the load carried the first thread of ISO Thread and 
ACME thread, and ACME thread and Square thread are 5\%-6\% and 3\%-4\%, respectively. The difference is independent of the size of the bolt. However, similar to the 2D simulation, the load distribution was affected by the size of the bolt.

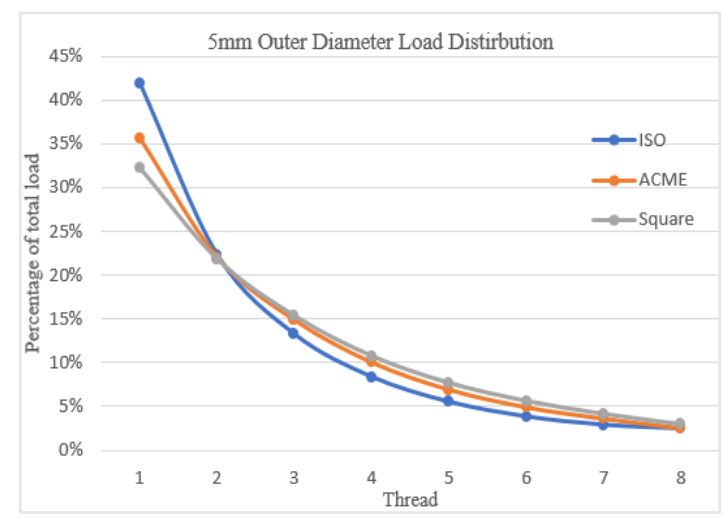

(a)

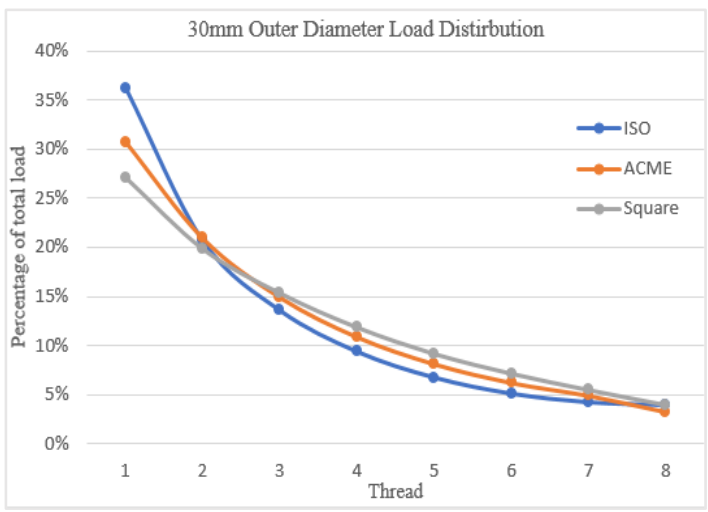

(b)

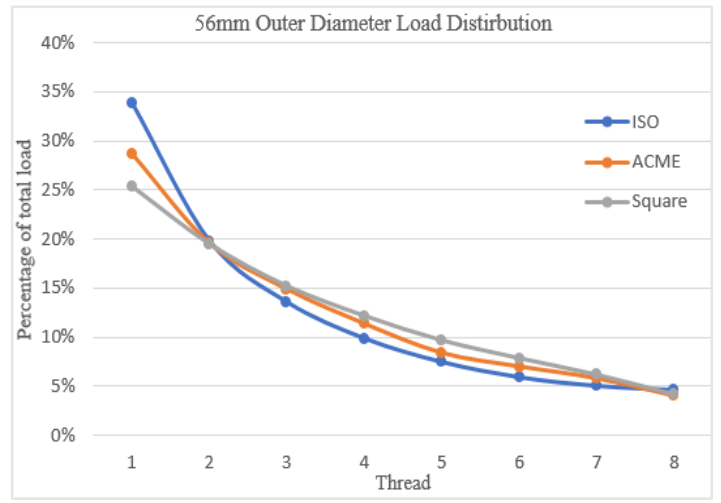

(c)

Figure 10. Effect of thread geometry on load distribution (3D), a) $D=5 \mathrm{~mm}, \mathrm{~b}) \mathrm{D}=30 \mathrm{~mm}, \mathrm{c}) \mathrm{D}=56 \mathrm{~mm}$.

Figure 11 illustrates the difference in load distribution behavior when the size is varied. The load carried by the first two threads is greatest for the $5 \mathrm{~mm}$ size, and lowest for the $56 \mathrm{~mm}$ size. The load on the third thread is approximately equal for the three sizes for the same thread geometry. The remainder of the force is distributed to the remaining threads. Although the difference for the load on the first thread of 4\%-6\% remains consistent for $5 \mathrm{~mm}$ to $30 \mathrm{~mm}$ size variation from the 2D simulation results, the difference for the $30 \mathrm{~mm}$ to $56 \mathrm{~mm}$ size variation is $2 \%-3 \%$, meaning that the difference in the load carried by the first thread decreases with the increase in size, but the differences are the same for the three thread geometries. The differences, however, are only true for the ISO Coarse series; but the trend itself is true since $\mathrm{D}$ and $\mathrm{P}$ always increase disproportionately for series of thread unless it is a custom size of thread. 


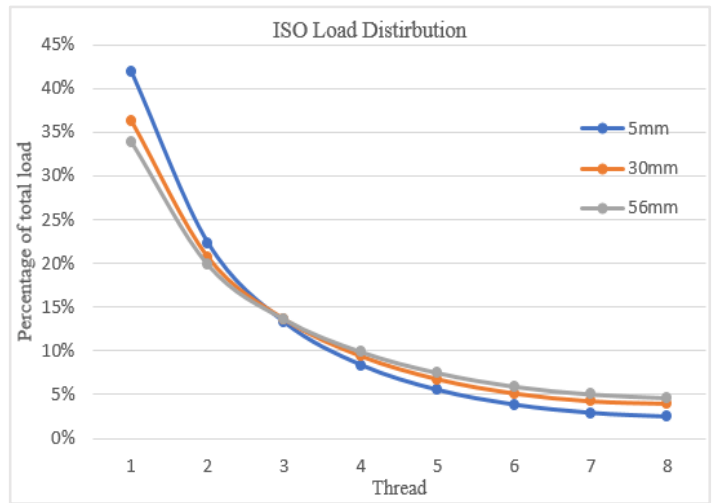

(a)

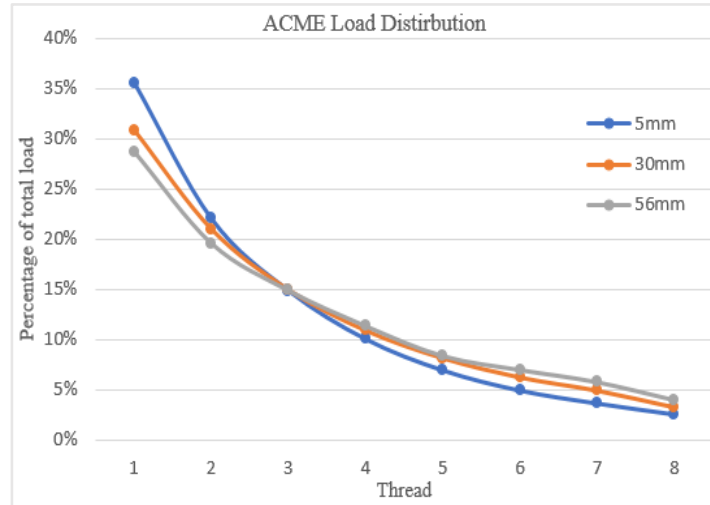

(b)

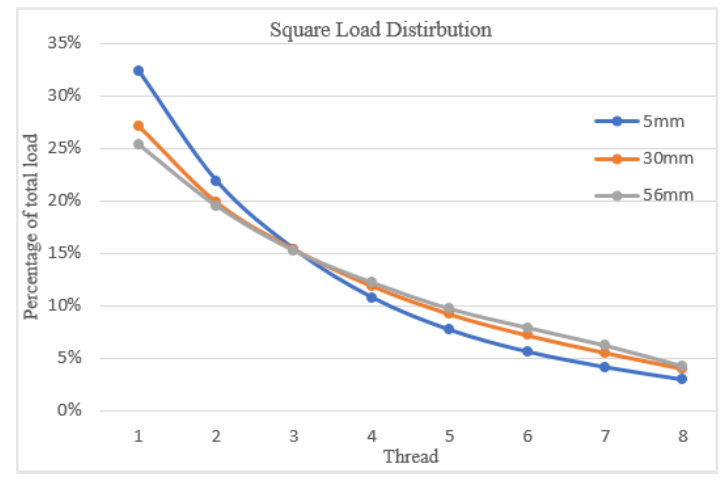

(c)

Figure 11. Effect of size on load distribution (3D), a) ISO, b) ACME, c) Square.

\section{CONCLUSION}

A numerical study is developed to investigate the effect of bolt thread geometry on the load distribution of continuous threads. In this regard, 2D and 3D simulations are conducted which unveiled novel relationships between thread parameters that were not studied of beforehand. These can be summarized as follow:

1. The first thread of the ISO bolt carries the most force, followed by ACME thread, and then Square thread carries the least compared to the other thread geometries of the same D and $\mathrm{P}$. The remainder of the load is spread out to the remaining threads.

2. The difference in the load carried by the first thread of the ISO, Square, or Acme thread of the same size remains the same regardless of the size.

3. The load on the first two threads decreases when the size of the bolt is increased assuming the same thread type is used. 
4. The difference in the load carried by the first two threads when the size increases, decreases as the compared sizes become larger.

The 2D finite element simulation results, although validated with previous research studies, were mostly inconclusive in determining the load distribution behavior when the thread geometry is changed. The 3D finite element simulations were more conclusive in determining the load distribution trends.

\section{ACKNOWLEDGMENT}

The authors acknowledge the financial support of the Ministry of Education, Youth and Sports of the Czech Republic and the European Union (European Structural and Investment Funds - Operational Programme Research, Development and Education) in the frames of the project "Modular platform for autonomous chassis of specialized electric vehicles for freight and equipment transportation”. Reg. No. CZ.02.1.01/0.0/0.0/16 025/0007293.

\section{REFERENCES}

Beardmore, R. (2013). Power Screws. (ROYMECH) Retrieved February 3, 2018, from http://www.roymech.co.uk/Useful_Tables/Cams_Springs/Power_Screws.html

Bretl, J. L., \& Cook, R. D. (1979). Modelling the load transfer in threaded connections by the finite element method. International Journal for Numerical Methods in Engineering, 14(9), 1359-1377.

Brutti C. (2017). Load and stress distribution in screw threads with modified washers. Journal of Multidisciplinary Engineering Science and Technology. 4(1):6523.

Carvill, J. (2015). Mechanical Engineer's Data Handbook. Kent: Elsevier Science.

Chen, J. J., \& Shih, Y. S. (1999). A study of the helical effect on the thread connection by three dimensional finite element analysis. Nuclear engineering and design, 191(2), 109-116.

Coria I, Abasolo M, Gutiérrez A, Aguirrebeitia J. (2020). Achieving uniform thread load distribution in bolted joints using different pitch values. Mechanics \& Industry. 21.

Den Hartog, J. P. (1929). The Mechanics of Plate Rotors for Turbogenerators. Trans. ASME, Paper no. APM S1$151,1-10$.

Dhoska, K. (2019). Tensile Testing Analysis of the HRB400 Steel Reinforcement Bar: Tensile Testing Analysis of the HRB400 Steel Reinforcement Bar. International Journal of Innovative Technology and Interdisciplinary Sciences, 2(3), 253-258. https://doi.org/10.15157/IJITIS.2019.2.3.253-258

Dragoni, E. (1994). Effect of thread pitch and frictional coefficient on the stress concentration in metric nut-bolt connections. Transactions-American society of Mechanical Engineers journal of offshore mechanics and Arctic Engineering, 116, 21-21.

Dragoni E (1997). Effect of thread pitch on the fatigue strength of steel bolts. Proceedings of the Institution of Mechanical Engineers, Part C: Journal of Mechanical Engineering Science.211(8):591-600.

Engineers Edge. (n.d.). ACME General Purpose Thread Design. (Engineers Edge) Retrieved February 10, 2018, from https://www.engineersedge.com/hardware/acme-threads-calculator.htm

Geramizadeh M, Katoozian H, Amid R, Kadkhodazadeh M. (2018). Three-dimensional optimization and sensitivity analysis of dental implant thread parameters using finite element analysis. Journal of the Korean Association of Oral and Maxillofacial Surgeons. 44(2):59. 
Goodier, J. N. (1940). The Distribution of Load on the Threads of Screws Trans. ASME, Journal of Applied Mechanics, 61.

Hetenyi, M. (1943). The distribution on of stress in threaded connections. Proc. SESA(1), 1.

ISO - International Organization for Standardization. (2014, April 11). ISO 68-1:1998: ISO general purpose screw threads -- Basic profile -- Part 1: Metric screw threads. (ISO) Retrieved November 28, 2017, from https://www.iso.org/standard/3707.html

Karimzadeh, A., Rahimian Koloor S.S., Ayatollahi M. R., Bushroa A. R., Yahya M. Y. (2019). Assessment of nano-indentation method in mechanical characterization of heterogeneous nanocomposite materials using experimental and computational approaches." Scientific reports 9, no. 1. 1-14.

Kenny, B., \& Patterson, E. A. (1985). Load and stress distribution in screw threads. Experimental Mechanics, 25(3), 208-213.

Koloor, S. S. R., A. Abdul-Latif, and Mohd Nasir Tamin. (2011). Mechanics of composite delamination under flexural loading. In Key Engineering Materials, vol. 462, pp. 726-731. Trans Tech Publications Ltd.

Koloor, S. S. R., M. A. Abdullah, M. N. Tamin, and M. R. Ayatollahi. (2019). Fatigue damage of cohesive interfaces in fiber-reinforced polymer composite laminates." Composites Science and Technology 183. 107779 .

Lee, J. E., Kim, J. B., Park, K., \& Ra, S. W. (2016). Investigation into thread rolling characteristics of sub miniature screws according to thread shapes. Transactions of the Korean Society of Mechanical Engineers, A, 40(11), 971-978. doi:10.3795/KSME-A.2016.40.11.971

Maruyama, K. (1974). Stress Analysis of a Bolt-Nut Joint by the Finite Element Method and the CopperElectroplating Method:(2nd Report, Stress at the Root of a Bolt Thread under a Tensile Load). Bulletin of JSME, 17(106), 442-450.

NarendraKumar U, Mathew AT, Iyer N, Rahman F, Manjubala I. (2018). A 3D finite element analysis of dental implants with varying thread angles. Materials Today: Proceedings. 5(5):11900-5.

Ng, Theng Pin, S. S. R. Koloor, J. R. P. Djuansjah, and MR Abdul Kadir. (2017). Assessment of compressive failure process of cortical bone materials using damage-based model. Journal of the mechanical behavior of biomedical materials 66: 1-11.

Nie X, Hu A, Huo J, Guo J. (2018). Load distribution analysis and experimental verification of TBM thread connection structure. In2018 3rd International Workshop on Materials Engineering and Computer Sciences (IWMECS 2018) pp. 367-375, Atlantis Press

Sato, T., Yamamoto, K., Li, R., \& Ando, H. (2008). Finite element analysis of load distribution of threaded connections made of dissimilar materials in high pressure facilities. ASME Pressure Vessels and Piping Conference, 2, 237-244.

Sopwith, D. G. (1948). The distribution of load in screw threads. Proceedings of the Institution of Mechanical Engineers, 159(1), 373-383.

Rahimian Koloor, S. S., Karimzadeh A., Noorfaizal Y., Petrů M., Ayatollahi M. R., and Mohd N. T. (2020). An energy-based concept for yielding of multidirectional FRP composite structures using a mesoscale lamina damage model. Polymers 12, no. 1. 157.

Rahimian Koloor, S. S., Karimzadeh A., Mohd N. T., Abd Shukor M. H. (2018). Effects of sample and indenter configurations of nanoindentation experiment on the mechanical behavior and properties of ductile materials." Metals 8, no. 6: 421. 
Tan, P. S., Akhavan Farid, A., Karimzadeh, A., Rahimian Koloor, S. S., \& Petrů, M. (2020). Investigation on the Curvature Correction Factor of Extension Spring. Materials, 13(18), 4199.

Wang, G., \& Ding, Y. (2020). The effects of uniform temperature and bolts fracture on the behaviour of high strength bolted frictional steel connection in dashegnguan railway bridge. Journal of Engineering Research (Kuwait), 8(1), 107-127. doi:10.36909/jer.v8i1.6500

Yamamoto, A. (1980). The Theory and Computation of Threads Connection (in Japanese). Yoken-do, Tokyo.

Yang, G., Hong, J., Zhu, L., Li, B., Xiong, M., \& Wang, F. (2013). Three-dimensional finite element analysis of the mechanical properties of helical thread connection. Chinese Journal of mechanical engineering, 26(3), 564-573.

Zhang, D., Gao, S., Niu, S., \& Liu, H. (2018). A Prediction Method for Load Distribution in Threaded Connections. Journal of Theoretical and Applied Mechanics (Poland), 56(1), 157-168.

Zhou W, Zhang R, Ai S, He R, Pei Y, Fang D. (2015). Load distribution in threads of porous metal-ceramic functionally graded composite joints subjected to thermomechanical loading. Composite Structures. 15;134:680-8. 